THE EUROPEAN ANAESTHESIOLOGY CONGRESS

\title{
EFFECTS OF EPIDURAL AND INTRAVENOUS REMIFENTANIL ANALGESIA DURING LABOR ON NEONATAL OUTCOME - RETROSPECTIVE OBSERVATIONAL STUDY
}

Sobot Novakovic $S^{1}$, Cuk $S^{1}$, Lazic $\mathbf{G}^{1}$, Rakanovic $D^{1}$, Banicevic Ceric $\mathrm{A}^{2}$, Visekruna $\mathrm{Lj}^{3}$

University Clinical Center of Republic of Srpska, Department of Anesthesia and Intensive Care

University Clinical Center of Republic of Srpska, Department of Gynecology and Obstetrics²

University Clinical Center of Republic of Srpska, Department of Pediatrics ${ }^{3}$

\section{BACKGROUND:}

It is widely assumed that any pharmacological form of analgesia must have adverse effects on the baby, while unmodified labor is relatively harmless. Reports have linked epidural with a variety of adverse effects during labor and labor outcome indirectly affecting neonatal outcome. Variety of neonatal side effects were reported including jaundice, sepsis, depressed neurobehavioral testing. Systemic analgesia with opioids has been connected with neonatal side effects as lower Apgar scores, depressed respiration, muscle tone and suckling and lower neurobehavioral scores.

\section{OBJECTIVE}

We evaluated the clinical outcome of term neonates bom to mothers who received epidural analgesia (E) or systemic analgesia with remifentanil ( $R$ ) during labor with aim to compare whether is one type of analgesia superior to another in term of neonatal outcome.

METHODS:

Data was collected retrospectively over the course of one year. We have evaluated the medical records of 247 full term neonates, 208 were bom to mothers who received $E$ and 39 to mothers who received R. Data on Apgar scores and perinatal complications (infection, sepsis, hyperbilirubinemia, perinatal injuries, asphyxia), and average hospital stay were collected. Mann-Whitney U test, chi square test, and logistic regression analysis were used where appropriate.

\section{RESULTS:}

Mean Apgar scores in 1st and 5th minute between $E$ and $R$ were similar ( 8.83 vs. 8.97 $P=0.252 ; 9.81$ vs. $9.87, P=0.762$, respectively). Average neonatal hospital stay was not different between groups ( 4.19 vs. $4, P=0.557$ ). The percentage of neonates with any perinatal complication were similar between groups $(28.3 \%$ vs. $32.5 \%, P=0.598)$. Neonates bom by cesarean delivery (CD) had statistically significant worse outcomes compared to neonates that were delivered vaginally (odds ratio $2.895 \% \mathrm{Cl}$ [1.30647-6.17692]).

\section{CONCLUSIONS:}

We did not find statistically significant difference in mean Apgar scores and perinatal complications between neonates who received epidural vs. remifentanil analgesia. We measured an increased rate of complications in neonates born via CD. Fetal indications for CD may be a significant confounder explaining this finding. Future studies should have a greater sample size and be powered to detect such associations.

Figure 1. Neonatal Complications Frequency Compared to Type of Delivery

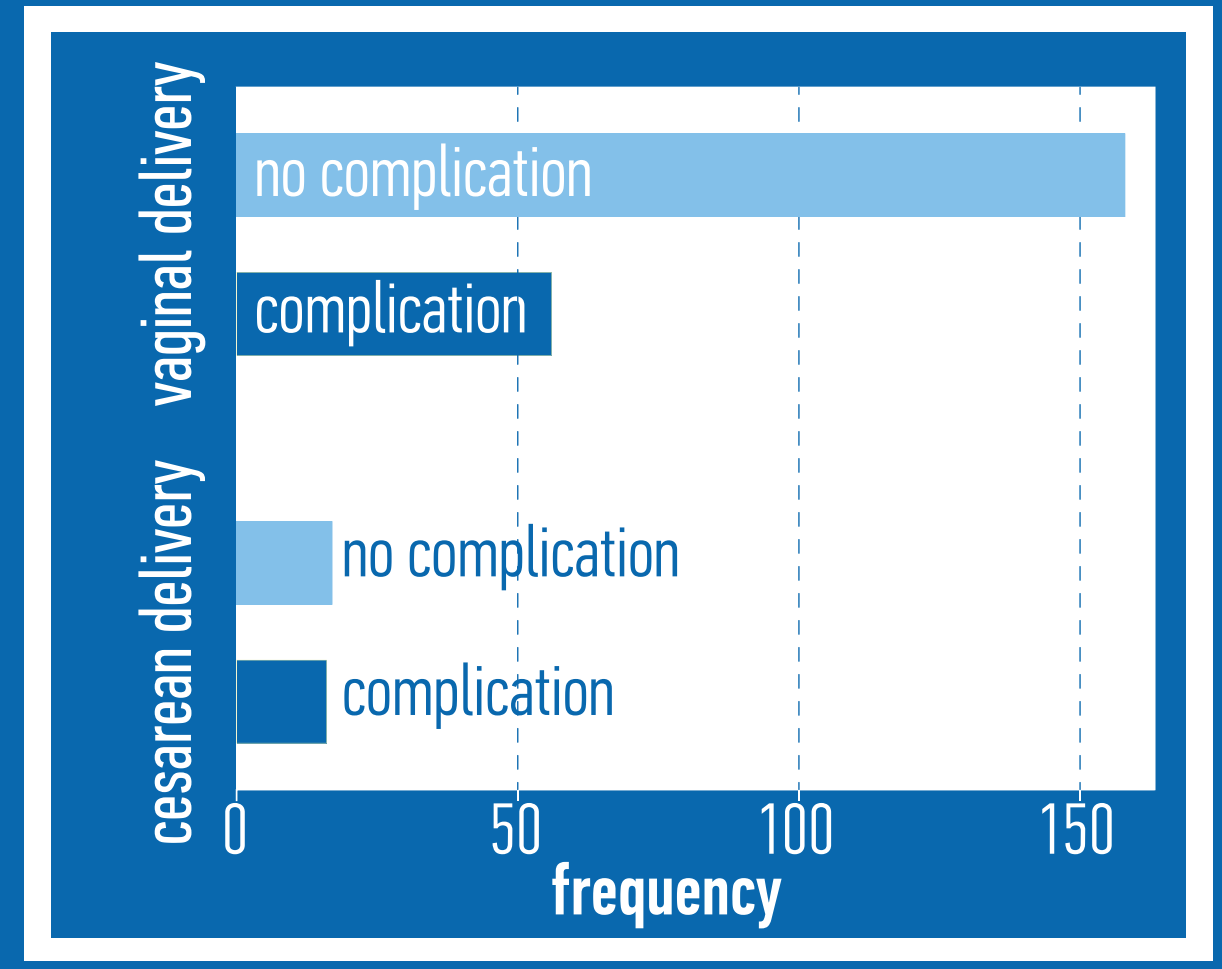

\section{Table 1. Maternal Demographic Characteristics}

\begin{tabular}{|cccc|}
\hline & Epidural $(\mathrm{n}=208)$ & Remifentanil $(\mathrm{n}=39)$ & P value \\
\hline Maternal age & 30.8 & 32.7 & 0.1354 \\
\hline Gestational week & 39.4 & 39.5 & 0.3635 \\
\hline Maternal diseases** & $31(14.9)$ & $8(20.51)$ & 0.378 \\
\hline Parity & & & \\
\hline Primipara & $135(64.9)$ & $29(74.36)$ & \multirow{2}{*}{0.251} \\
\hline Multipara & $73(35.1)$ & $10(25.64)$ & \\
\hline
\end{tabular}

*Data presented as mean, $\mathrm{n}(\%)$

**Maternal diseases included thrombophilia, PIH, preeclampsia, hypothyroidism, hyperthyroidism, thrombocytopenia, cardiac disease

Table 2. Neonatal Demographic Characteristic and Neonatal Outcomes

\begin{tabular}{|c|c|c|c|c|}
\hline & & Epidural (N=208) & $\begin{array}{l}\text { Remifentanil } \\
\quad(\mathrm{N}=39)\end{array}$ & P value*** \\
\hline \multicolumn{2}{|l|}{ Birth weight } & 3563.3 & 3506.9 & $0.3620^{x}$ \\
\hline \multicolumn{2}{|l|}{ Birth length } & 52.8 & 52.5 & $0.3735^{x}$ \\
\hline \multicolumn{5}{|l|}{ Apgar score } \\
\hline \multicolumn{2}{|l|}{$1 \mathrm{~min}$} & 8.83 & 8.97 & $0.2523 \dagger$ \\
\hline \multicolumn{2}{|l|}{$5 \mathrm{~min}$} & 9.81 & 9.87 & $0.7620 \dagger$ \\
\hline \multicolumn{2}{|c|}{ Apgar score $<7$ in 1st min } & 3 & 0 & $N C^{* * * *}$ \\
\hline \multicolumn{2}{|c|}{ Apgar score $<7$ in 5th min } & 1 & 0 & $N C^{* \star * \star}$ \\
\hline \multicolumn{2}{|c|}{ Neonatal Perinatal Complications* } & $59(28.3)$ & $13(32.5)$ & $0.598 \neq$ \\
\hline \multicolumn{2}{|l|}{ Infections } & $47(22.6)$ & $8(20.5)$ & $0.774 \ddagger$ \\
\hline \multicolumn{2}{|l|}{ Hyperbilirubinemia } & $10(5.13)$ & $2(4.8)$ & $0.932 \ddagger$ \\
\hline \multicolumn{2}{|l|}{ Injuries } & $7(3.3)$ & $1(2.5)$ & $N C^{* * * *}$ \\
\hline \multicolumn{2}{|c|}{ Intracranial hemorrhage and ischemia } & 2 & 1 & $N C^{* * * *}$ \\
\hline \multicolumn{2}{|l|}{ Respiratory distress } & 1 & 0 & $N C^{* * * *}$ \\
\hline \multicolumn{2}{|l|}{ Admission to NICU } & 5 & 0 & $N C^{* * * *}$ \\
\hline \multicolumn{2}{|c|}{ Admission to observational neonatal unit } & $15(7.2)$ & $1(2.5)$ & $0.279 \ddagger$ \\
\hline \multicolumn{2}{|c|}{ Average hospital stay } & 4.2 & 4 & $0.5571 \dagger$ \\
\hline \multicolumn{5}{|c|}{$\begin{array}{l}\text { *Perinatal complications include infections, hyperbilirubinemia, injuries, perinatal asphyxia } \\
{ }^{* \star D} \text { ata presented as mean, } n(\%) \\
{ }^{* \star *} \text { values calculated with Student t-testx, Mann-Whitneył and Chi squared test } \\
{ }^{* \star \star N C}-\text { not able to calculate }\end{array}$} \\
\hline \multicolumn{5}{|l|}{ Table 3. Labor Outcomes } \\
\hline & \multicolumn{2}{|c|}{ Epidural (N=208) } & entanil (N=39) & $P$ value** \\
\hline Labor duration (min) & \multicolumn{2}{|c|}{359.5} & 317.1 & $0.3752 \dagger$ \\
\hline Vaginal delivery & \multicolumn{2}{|c|}{$181(86.2)$} & $35(89.8)$ & \multirow{2}{*}{$0.535 \ddagger$} \\
\hline Conversion to $C$ section & \multicolumn{2}{|c|}{$28(13.8)$} & $4(10.2)$ & \\
\hline \multicolumn{5}{|l|}{ Indication for $C$ section } \\
\hline Fetal distress & \multicolumn{2}{|c|}{$9(4.33)$} & $3(7.69)$ & $0.000 \ddagger$ \\
\hline Prolonged labor & \multicolumn{2}{|c|}{$11(5.29)$} & $1(2.56)$ & $0.111 \neq$ \\
\hline Cephalopelvic disproportion & \multicolumn{2}{|c|}{$8(3.85)$} & 0 & $N C^{* * *}$ \\
\hline \multicolumn{5}{|c|}{$\begin{array}{l}\text { *Data presented as mean, } n(\%) \\
\text { D*x values calculated with Mann-Whitney test }+ \text { and chi squared testł } \\
\text { ***NC - not able to calculate }\end{array}$} \\
\hline
\end{tabular}

